\title{
DFMG reverses proliferation and migration of vascular smooth muscle cells induced by co-culture with injured vascular endothelial cells via suppression of the TLR4-mediated signaling pathway
}

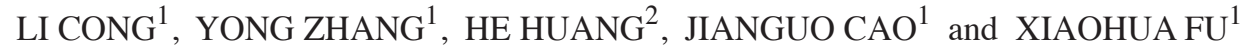 \\ ${ }^{1}$ Medical College, Hunan Normal University, Changsha, Hunan 410013; ${ }^{2}$ Department of Pathology, \\ Maternal and Child Health Hospital of Hunan Province, Changsha, Hunan 410008, P.R. China
}

Received August 8,2017; Accepted December 13, 2017

DOI: $10.3892 / \mathrm{mmr} .2018 .8635$

\begin{abstract}
Difluoromethoxy-5,4'-dimethoxy-genistein (DFMG) is a novel chemical compound synthesized using genistein. Previous studies have indicated that DFMG can reverse the apoptosis of vascular endothelial cells (VECs) by regulating the mitochondrial apoptosis pathway. The present study aimed to investigate the activity and molecular mechanism underlying DFMG-mediated protection of vascular smooth muscle cell (VSMCs) using a non-contact co-culture model established by using Transwell insert. Secretion of interleukin-6 (IL-6) and tumor necrosis factor- $\alpha$ (TNF- $\alpha$ ) were measured by ELISA. Proliferation and migration of VSMCs were assessed using a Cell Counting kit- 8 and wound healing assays, respectively. Toll-like receptor 4 (TLR4) mRNA and protein levels were detected by reverse transcription-quantitative polymerase chain reaction and western blotting analyses, respectively. In the present study, lysophosphatidylcholine (LPC) significantly increased the secretion of IL-6 and TNF- $\alpha$ in VECs. VECs treated with LPC markedly increased proliferation and migration of VSMCs, which were inhibited by DFMG. Transfection of either TLR4 short hairpin RNA (shRNA) or TLR4 cDNA in VECs inhibited and increased proliferation and migration of VSMCs, respectively. Furthermore, transfection of VECs with
\end{abstract}

Correspondence to: Dr Xiaohua Fu, Medical College, Hunan Normal University, 371 Tongzipo Road, Changsha, Hunan 410013, P.R. China

E-mail: fuxh1@126.com

Abbreviations: AS, atherosclerosis; DFMG, 7-difluoromethoxy5,4'-dimethoxy-genistein; IL-6, interleukin-6; LPC, lysophosphatidylcholine; TLR4, Toll-like receptor 4; TNF- $\alpha$, tumor necrosis factor- $\alpha$; VECs, vascular endothelial cells; VSMCs, vascular smooth muscle cells

Key words: 7-difluoromethoxy-5,4'-dimethoxy-genistein, TLR4, vascular smooth muscle cells, vascular endothelia cells, co-culture of cells, proliferation, migration
TLR4 shRNA suppressed the proliferation and migration of VSMCs induced by co-culture with injured VECs, which was further enhanced by treatment with DFMG. By contrast, transfection of VECs with TLR4 cDNA enhanced proliferation and migration of VSMCs and this effect was inhibited by treatment with DFMG. Taken together, the results of the present study demonstrated that DFMG can reverse proliferation and migration of VSMCs induced by co-culture with injured VECs via suppression of the TLR4-mediated signaling pathway.

\section{Introduction}

Vascular smooth muscle cells (VSMCs) are cellular components of the medial layer of the artery wall adjacent to vascular endothelial cells (VECs). VSMCs serve a number of roles under normal physiological conditions $(1,2)$. Proliferation and migration of VSMCs occurs during the early stage of atherosclerotic lesion formation and post angioplasty restenosis (3). Numerous cell types are involved in atherogenesis and a complex network of transcription factors and proteins is involved in this process (4).

VECs can regulate the contractile phenotype of VSMCs through modulation of miR-206/ADP-ribosylation factor 6 and sodium/calcium exchanger 1/exosome (2). When stimulated by endothelial injury signals, VSMCs migrate into intima and undergo a proliferative phenotypic switch (5). Macrophages contribute to pathogenesis of restenosis via secretion of growth factors, cytokines and chemokines, which induce proliferation and activation of VSMCs (4). Endothelial dysfunction is an early contributor and predictor of atherosclerosis (AS) $(6,7)$. Endothelial dysfunction results in the earliest detectable alterations during the development of atherosclerotic lesions $(6,7)$. The VECs-VSMCs co-culture model has been widely adopted for the investigation of AS $(2,8,9)$.

Data presented by a previous study supports the hypothesis that inflammation contributes to the initiation, progression and plaque rupture of AS (10). AS is dependent on innate immune responses and involves activation of Toll-like receptors (TLRs). TLRs are pattern-recognition receptors and a part of the interleukin-1 receptor/Toll-like receptor superfamily. Activation of TLRs leads to expression of inflammatory proteins that may 
lead to acute coronary syndrome (11-13). Different subtypes of TLRs are involved in a number of aspects of the inflammatory response.

TLR4 exhibits a high level of expression in aortic tissues from patients who have undergone coronary artery bypass graft surgery and is positively correlated with Gensini score (14). Kapelouzou et al (15) demonstrated that the TLR4 mRNA expression level was markedly upregulated in a hyperlipidemic rabbit model and was associated with the progression of AS. Studies in knockout mice demonstrated that the TLR4/nuclear factor $-\kappa \mathrm{B}(\mathrm{NF}-\kappa \mathrm{B})$ signaling pathway contributes to chronic unpredictable mild stress-induced AS via activation of pro-inflammatory cytokines (16). The TLR4/NF- $\kappa$ B signaling pathway also contributes to the early stage intimal foam cell accumulation at lesion-prone aortic sites (12). In vitro, TLR4 mediates hyperglycemia-induced inflammation and dysregulation of the endothelial glycocalyx levels in human macrovascular aortic ECs (17). TLR4 inhibitor CLI-095 can suppress the progression of AS by reducing macrophage foam cell formation (18). Yang et al (19) hypothesized that TLR4 signaling promoted a pro-inflammatory phenotype in VSMCs. Positive feedback regulation of VSMCs proliferation is mediated by the TLR4/Rac family small GTPase 1/Akt serine/threonine kinase signaling pathway (20). Furthermore, the TLR4/NF- $\mathrm{BB}$ signaling pathway can increase the expression of matrix metalloproteinase- $9 \mathrm{mRNA}$ and protein levels in order to stimulate migration of human aortic VSMCs (21).

7-Difluoromethoxy-5,4'-dimethoxy-genistein (DFMG) is a novel active chemical molecule. Our previous studies indicated that DFMG was more efficient compared with genistein (GEN) in reducing the risk of cardiovascular disease by inhibition of oxidative damage and/or inhibition of theTLR4 signaling pathway $(22,23)$. The authors of the present study, also previously reported that DFMG could protect VECs through inhibition of the mitochondrial apoptotic pathway $(24,25)$. Furthermore, the preventive effect of DFMG was more apparent compared with the therapeutic effect within apolipoprotein $\mathrm{E}^{-/}$mice induced by a cholesterol-rich diet (26).

The present study investigated whether TLR4 has a role in abnormal proliferation and migration of VSMCs induced by lysophosphatidylcholine (LPC)-injured VECs. The mechanism underlying DFMG-mediated protection of VSMCs from VECs-stimulated dysfunction in vitro has also been investigated. The present study aimed to determine the activity of DFMG on the proliferation and migration of VSMCs using a non-contact co-culture model with LPC-injured VECs.

\section{Materials and methods}

Reagents and plasmids. LPC was purchased from Sigma-Aldrich (Merck KGaA, Darmstadt, Germany) and dissolved in PBS. DFMG (purity, >99\%) was synthesized as previously described (27) and dissolved in dimethyl sulfoxide (Amresco, LLC, Solon, OH, USA). DFMG was subsequently filter-sterilized. Human interleukin-6 (IL-6) and tumor necrosis factor- $\alpha$ (TNF- $\alpha$ ) ELISA kits were purchased from Shanghai ExCell Biology, Inc. (EH004-96 and EH009-96; Shanghai, China). The Cell Counting kit-8 (CCK-8) was purchased from Vazyme (Piscataway, NJ, USA). MegaTran1.0 transfection reagent, p-green fluorescent protein (GFP)-V-RS-TLR4-short hairpin (sh)RNA and pCMV6-AC-GFP-TLR4-cDNA plasmids were obtained from OriGene Technologies, Inc. (Rockville, MD, USA). Anti-TLR4 and anti- $\beta$-actin antibodies were obtained from Abgent, Inc. (ASC10194 and AM1021B, San Diego, CA, USA). All secondary antibodies were supplied by ComWin Biotech Co. Ltd. (CW0156S and CW0102S; Beijing, China).

Cell culture. Human umbilical vein EC and human aortic SMC cell lines were obtained from the China Center for Type Culture Collection (Wuhan, China). Both cell lines were incubated in a humidified incubator at $37^{\circ} \mathrm{C}$ with $5 \% \mathrm{CO}_{2}$ and cultured in Dulbecco's modified Eagle's medium (DMEM), supplemented with $10 \%$ fetal bovine serum (FBS), $100 \mathrm{IU} / \mathrm{ml}$ penicillin $\mathrm{G}$ and $100 \mu \mathrm{g} / \mathrm{ml}$ streptomycin. Cell culture reagents were obtained from Biological Industries Israel Beit-Haemek Ltd. (Beit HaEmek, Israel).

Determination of IL- 6 and TNF- $\alpha$ levels. VECs were pretreated with $\operatorname{LPC}(10,20,30,40$ and $50 \mu \mathrm{M})$ at $37^{\circ} \mathrm{C}$ for $24 \mathrm{~h}$; the levels of IL-6 and TNF- $\alpha$ in supernatants were quantified using human IL- 6 and TNF- $\alpha$ ELISA kits (aforementioned) according to the manufacturer's protocol. A total of $100 \mu \mathrm{l}$ serially-diluted standard samples and/or supernatant samples were added into the microplate and incubated at $37^{\circ} \mathrm{C}$ for $1 \mathrm{~h}$. The plate was further incubated with $100 \mu \mathrm{l}$ antibody solution against IL- 6 or TNF- $\alpha$ at $37^{\circ} \mathrm{C}$ for an additional $1 \mathrm{~h}$. Subsequently, $100 \mu \mathrm{l}$ horseradish peroxidase-conjugated secondary antibodies were added and the plate was incubated at $37^{\circ} \mathrm{C}$ for $30 \mathrm{~min}$. The samples were washed four times with $100 \mu \mathrm{l}$ PBS containing Tween-20 (PBST) prior to incubation with secondary antibodies. Finally, the plates were incubated with $100 \mu \mathrm{l}$ substrate in the dark at $37^{\circ} \mathrm{C}$ for $15 \mathrm{~min}$. The absorbance was measured at a wavelength of $450 \mathrm{~nm}$ with a spectrophotometer (Bio-Rad Laboratories, Inc., Hercules, CA, USA).

Establishment of the co-culture model. Co-culture of VECs and VSMCs was performed using a Transwell chamber with a $10 \mu \mathrm{m}$-thick porous membrane. The membrane contained $0.4 \mu \mathrm{m}$ pores in a Transwell cell culture insert (Corning Incorporated, Corning, NY, USA). This prevented cells from direct contact. Briefly, VSMCs $\left(1 \times 10^{5}\right.$ cells/well) were seeded in 6-well plates and cultured for $24 \mathrm{~h}$ prior to co-culture with pretreated and/or transfected VECs. VECs $\left(1 \times 10^{5}\right.$ cells/well $)$ were individually cultured on the insert filters for $24 \mathrm{~h}$, then pretreated with DFMG $(0.3,1.0$ and $3.0 \mu \mathrm{M})$ for 12 or $24 \mathrm{~h}$ prior to treatment with LPC for $24 \mathrm{~h}$, or pretreated following transfection for $48 \mathrm{~h}$. Subsequently, VECs $\left(1 \times 10^{5}\right.$ cells/well $)$ with inserts were added to the upper chamber of the Transwell system (VSMCs at the bottom). Both cells were maintained in DMEM with $10 \% \mathrm{FBS}$ at $37^{\circ} \mathrm{C}$ for $24 \mathrm{~h}$. A light microscope was used to observe migratory cells in lower chamber; magnification, $\mathrm{x} 200$.

VSMCs viability assay. The CCK-8 assay was used in order to detect cell viability of VSMCs. VSMCs were co-cultured with VECs treated with different interventions for $24 \mathrm{~h}$. VSMCs were digested and cultured in $100 \mu 1$ medium in 96-well plates $\left(1 \times 10^{4}\right.$ cells/well) with four replicates for $24 \mathrm{~h}$. Subsequently, 
the medium was replaced and $10 \mu \mathrm{lCCK}-8$ solution was added to each well for an additional $2 \mathrm{~h}$ incubation at $37^{\circ} \mathrm{C}$. The absorbance was measured at a wavelength of $450 \mathrm{~nm}$. Cell viability was calculated based on the optical density values of each group.

VSMCs wound healing (migration) assay. The wound healing assay was carried out as previously described (28). Prior to co-culture, VSMCs were seeded on 6-well plates and scratched using a $200 \mu \mathrm{l}$ pipette tip in order to generate a cell-free area. PBS was used to rinse cell debris three times and cells were incubated in FBS-free media in order to prevent proliferation. Phase contrast images of cells were captured at 0 h. Furthermore, pretreated and/or transfected VECs with inserts were added to the upper chamber of the Transwell system. Following $24 \mathrm{~h}$ incubation at $37^{\circ} \mathrm{C}$, images of the same plates and cells were captured. Cell-free areas were quantified using Adobe Photoshop CS6 software (Adobe Systems, Inc., San Jose, CA, USA). The migration was expressed as percentage coverage.

VECs transfection. Transfection of VECs with TLR4 shRNA and cDNA was performed out MegaTran 1.0, a non-lipid polymer-based transfection reagent with relatively low toxicity on the transfected cells. VECs were seeded on 6-well plates and cultured until $50-70 \%$ confluence prior to transfection. $3 \mu \mathrm{g}$ TLR4 shRNA, cDNA and corresponding negative control (only with GFP gene) plasmid ( $3 \mu \mathrm{g}$ ) was diluted in $300 \mu \mathrm{l}$ DMEM and vortexed gently. A total of $9 \mu 1$ MegaTran 1.0 was added to the diluted DNA and vortexed immediately for $10 \mathrm{sec}$. Samples were incubated for $10 \mathrm{~min}$ at room temperature and the MegaTran 1.0/DNA mixture was added to each well (already containing $2 \mathrm{ml}$ DMEM). The plate was shaken gently in order to achieve an even distribution of the complexes and incubated at $37^{\circ} \mathrm{C}$ for $4 \mathrm{~h}$. The expression of TLR4 was detected at 24 to $48 \mathrm{~h}$ following transfection.

RNA isolation and reverse transcription-quantitative polymerase chain reaction ( $R T-q P C R)$. Total RNA was extracted from the cells using TRIzol reagent (Invitrogen; Thermo Fisher Scientific, Inc., Waltham, MA, USA), according to the manufacturer's protocol. To quantify the mRNA, $500 \mathrm{ng}$ RNA from each sample were reverse transcribed into complementary DNA (cDNA) at $50^{\circ} \mathrm{C}$ for $15 \mathrm{~min}$, then $85^{\circ} \mathrm{C}$ for $5 \mathrm{sec}$ using HiScript Q RT SuperMix for qPCR (Vazyme Biotech Co., Ltd., Nanjing, China). qPCR was carried out using SYBR Premix ExTaq ${ }^{\mathrm{TM}}$ (Vazyme) and a 7500 fast qPCR system (Applied Biosystems; Thermo Fisher Scientific, Inc.). Cycling conditions consisted of an initial incubation at $95^{\circ} \mathrm{C}$ for $5 \mathrm{~min}$, then 40 cycles of $10 \mathrm{sec}$ at $95^{\circ} \mathrm{C}$ and $30 \mathrm{sec}$ at $60^{\circ} \mathrm{C}$, final extension for $15 \mathrm{sec}$ at $95^{\circ} \mathrm{C}$ and $1 \mathrm{~min}$ at $60^{\circ} \mathrm{C}$. The samples were run in triplicate. The $2^{-\Delta \Delta \mathrm{Cq}}$ method was used to analyze relative gene expression (29). GAPDH was used as an endogenous housekeeping gene control. The qPCR primer sequences are listed in Table I.

Western blotting. Cells were digested and subsequently lysed on ice using radioimmunoprecipitation assay lysis buffer containing $1 \%$ phenylmethyl sulfonyl fluoride. Samples were centrifuged at $12,000 \times \mathrm{g}$ at $4^{\circ} \mathrm{C}$ for $15 \mathrm{~min}$. Protein
Table I. Primer sequences used for qPCR.

\begin{tabular}{llc}
\hline Gene & \multicolumn{1}{c}{ Primer sequence $\left(5^{\prime}-3^{\prime}\right)$} & $\begin{array}{c}\text { Product } \\
\text { size }(\mathrm{bp})\end{array}$ \\
\hline GAPDH & $\begin{array}{l}\text { F: CAGGAGGCATTGCTGATGAT } \\
\text { R: GAAGGCTGGGGCTCATTT }\end{array}$ & 138 \\
TLR4 & $\begin{array}{l}\text { F: CCGAGGCCATTATGCTATGT } \\
\text { R: TCCCTTCCTCCTTTTCCCTA }\end{array}$ & 141 \\
& & \\
\hline
\end{tabular}

concentration was determined with a Bicinchoninic Acid protein assay kit (Beijing Solario Science \& Technology Co., Ltd., Beijing, China). A total of $25 \mu \mathrm{g}$ of each protein lysate was separated by $10 \%$ SDA-PAGE and transferred onto polyvinylidene difluoride membranes (EMD Millipore, Billerica, MA, USA) and blocked with 5\% skimmed milk in PBST (PBS containing $0.05 \%$ Tween-20) at $37^{\circ} \mathrm{C}$ for $1 \mathrm{~h}$. The membranes were incubated with rabbit anti-TLR4 antibody (1:1,000 dilution) and mouse anti- $\beta$-actin antibody $(1: 10,000$ dilution) on a shaking platform at $4^{\circ} \mathrm{C}$ overnight. Following washing of the membranes with PBST, goat anti-rabbit antibody (1:10,000 dilution) and goat anti-mouse antibody (1:10,000 dilution) were incubated with the membranes for $1 \mathrm{~h}$ at room temperature. The membranes were washed with PBST three times and protein expression was visualized by Pierce ECL Western Blotting Substrate (Pierce; Thermo Fisher Scientific, Inc.). The protein expression was quantified using densitometry and analyzed using GelPro software (version 3.2; Media Cybenetics, Inc., Rockville, MD, USA). $\beta$-actin expression was used as an internal control.

Statistical analysis. Data are presented as the mean \pm standard deviation from three independent experiments. SPSS software (version 17.0; SPSS, Inc., Chicago, IL, USA) and Graph Pad Prism 5 (GraphPad Software, Inc., La Jolla, CA, USA) were used for all statistical analyses. Differences between groups were examined by Student's t-test for two groups and one-way analysis of variance followed by a least significant difference test for multiple comparisons. $\mathrm{P}<0.05$ was considered to indicate a statistically significant difference.

\section{Results}

Establishment of an injured VECs-VSMCs co-culture model. Initially different concentrations of LPC were used in order to treat VECs and detect the secretion of IL-6 and TNF- $\alpha$. The results indicated that LPC induced VECs injury in a dose-dependent manner. LPC (20-50 $\mu \mathrm{M})$ significantly promoted the secretion of IL-6 and TNF- $\alpha$ (Fig. 1A and B). An increase in proliferation and migration of VSMCs was observed that was caused by co-culture with LPC-injured VECs (Fig. 1C-E). Consequently, $30 \mu \mathrm{M}$ LPC was selected in order to produce the injured VECs-VSMCs co-culture model.

$D F M G$ reverses the proliferation and migration of VSMCs induced by co-culture with injured VECs. To determine whether DFMG protects against proliferation and migration of VSMCs induced by co-culture with LPC-injured VECs, 
A
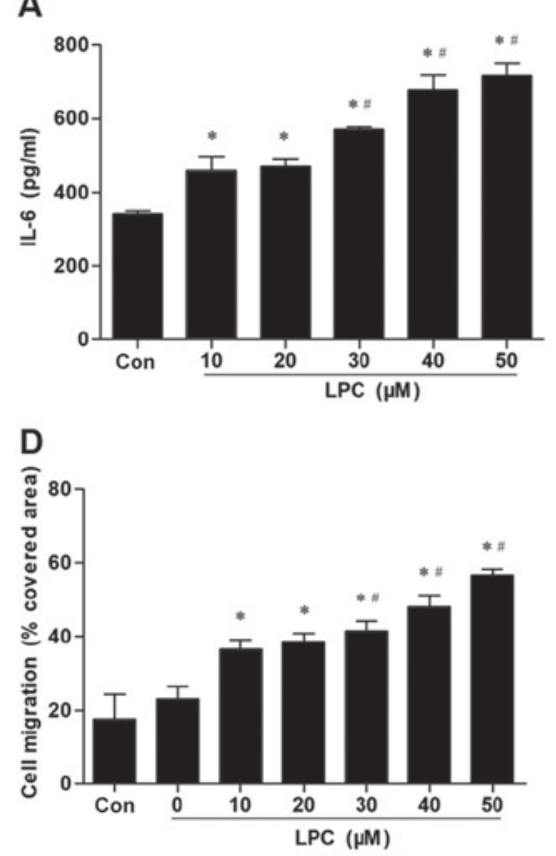

B

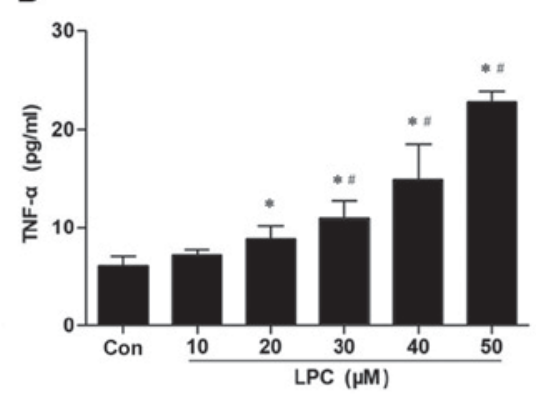

C

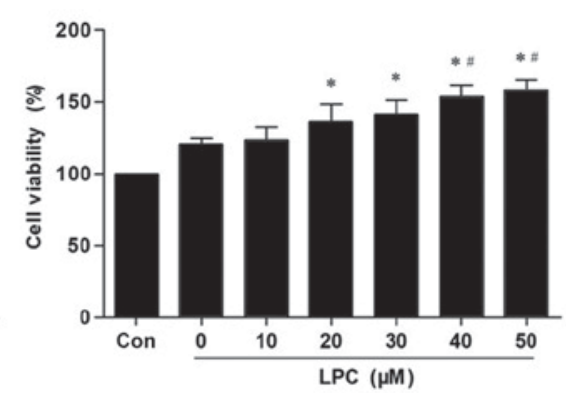

E

LPC $(\mu \mathrm{M})$

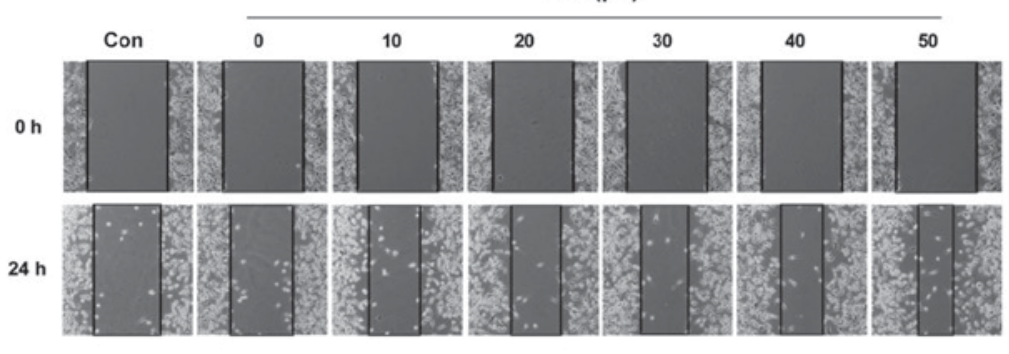

Figure 1. Effect of LPC on secretion of IL-6 and TNF- $\alpha$ in VECs, and proliferation and migration of VSMCs induced by co-culture with injured VECs. LPC $(20,30,40$ and $50 \mu \mathrm{M})$ significantly increased the levels of (A) IL-6 and (B) TNF- $\alpha$ in VECs-conditioned medium, in a concentration-dependent manner. Pre-treatment of VECs with LPC (20,30, 40 and $50 \mu \mathrm{M})$ significantly enhanced VMSC (C) survival and (D) migratory ability demonstrated by (E) wound healing induced by co-culture with injured VECs. Data are presented as the mean \pm standard deviation of three independent experiments. ${ }^{*}<0.05$ vs. Con. ${ }^{\#} \mathrm{P}<0.05$ vs. 10 and $20 \mu \mathrm{M}$ LPC. LPC, lysophosphatidylcholine; IL, interleukin; TNF- $\alpha$, tumor necrosis factor- $\alpha$; Con, control.

VECs were pretreated with various concentrations of DFMG for various durations prior to treatment with LPC $(30 \mu \mathrm{M})$. A significant decrease in the proliferation and migration of VSMCs was observed (Fig. 2). Proliferation and migration of VSMCs decreased following treatment with $\geq 1.0 \mu \mathrm{M}$ DFMG (Fig. 2A and C). Cell viability of the control group was set as $100 \%$. VECs were pretreated with $1.0 \mu \mathrm{M}$ DFMG and the relative cell viability of VSMCs was $\sim 86.7 \%$, whereas the corresponding healing rate was $\sim 30.73 \%$ (Fig. $2 \mathrm{~A}$ and C). Proliferation and migration of VSMCs was markedly reduced following pretreatment of VECs for $12 \mathrm{~h}$ with 1.0 or $3.0 \mu \mathrm{M}$ DFMG (Fig. 2B and D). The longer incubation time (24 h) demonstrated no significant effect on migration and proliferation of VSMCs (Fig. 2B and D). Thus, 1.0 $\mu \mathrm{M}$ DFMG was selected as the optimum concentration for the pretreatment of VECs for $12 \mathrm{~h}$.

Proliferation and migration of VSMCs co-cultured with VECs is inhibited by transfection with TLR4 shRNA. To further investigate the role of TLR4, transfection of TLR4 shRNA was used in order to silence TLR4 gene expression in VECs. VECs transfected with TLR4 shRNA exhibited significantly decreased expression of TLR4 mRNA and protein levels compared with those transfected with the GFP gene (Fig. 3A-C). TLR4 shRNA significantly inhibited the proliferation and migration of VSMCs co-cultured with VECs compared with the GFP control (Fig. 3D-F).

Proliferation and migration of VSMCs co-cultured with LPC-injured VECs is inhibited by DFMG treatment and TLR4 shRNA transfection. The effects of DFMG in combination with TLR4 shRNA transfection of VECs were investigated in order to determine whether DFMG could reverse the effects of LPC-injured VECs on the proliferation and migration of VSMCs. The results indicated that DFMG and TLR4 shRNA suppressed proliferation and migration of VSMCs, and the combination of these interventions was more effective than TLR4 knockdown alone (Fig. 4). DFMG and TLR4 shRNA exhibited a synergistic effect in promoting the stability of VSMCs.

TLR4 overexpression in VECs promotes proliferation and migration of VSMCs induced by co-culture with VECs. TLR4 cDNA significantly increased the expression of TLR4 mRNA and protein in VECs compared with the cells transfected with the GFP gene (Fig. 5A-C). Furthermore, transfection of the cells with TLR4 cDNA significantly enhanced the proliferation and migration of VSMCs in the co-culture model compared with the GFP control (Fig. 5D-F).

TLR4 cDNA transfection of VECs enhances injured $V E C$-induced proliferation and migration of VSMCs, which in turn attenuates the protective effect of DFMG. Combined treatment with DFMG and TLR4 cDNA transfection of VECs was performed in order to investigate whether TLR4 was involved in the DFMG mediated anti-migratory and anti-proliferative effect in VSMCs. The results indicated that TLR4 cDNA transfection significantly attenuated the inhibitory effects of DFMG on the proliferation and migration of VSMCs (Fig. 6).

\section{Discussion}

During the pathogenesis of atherosclerosis, the endothelial dysfunction is considered to be an initial step. Various risk 
A

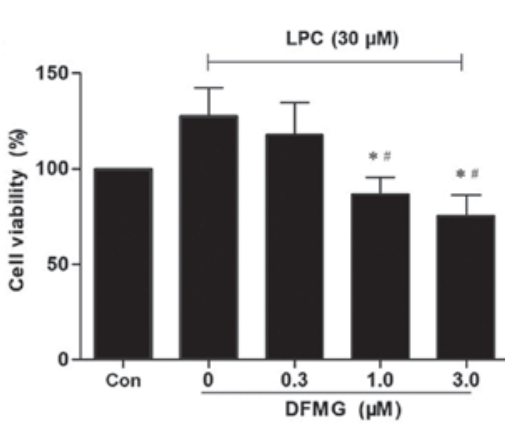

C
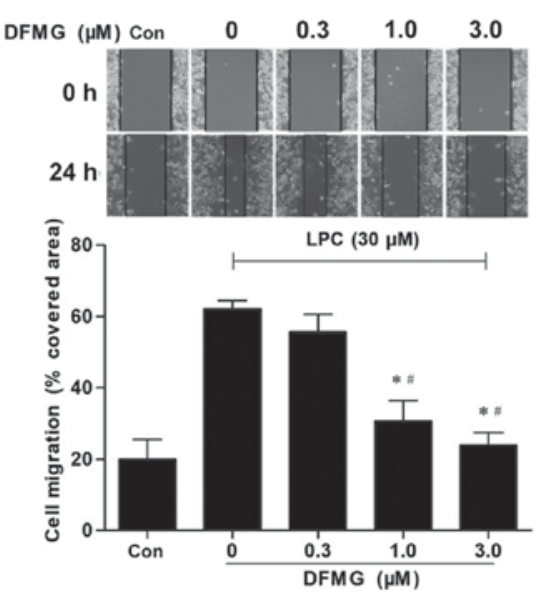

B

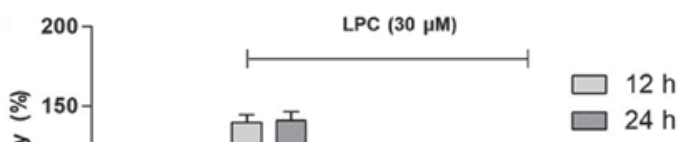

$24 \mathrm{~h}$

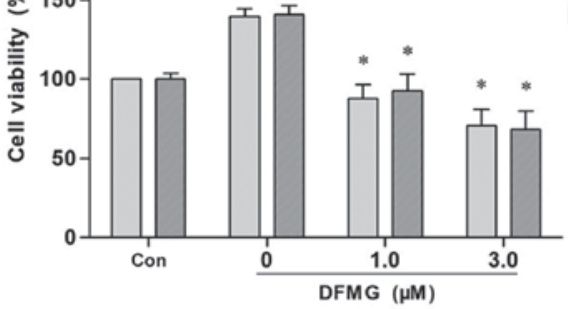

D DFMG ( $\mu$ M) Con

0

1.0

3.0
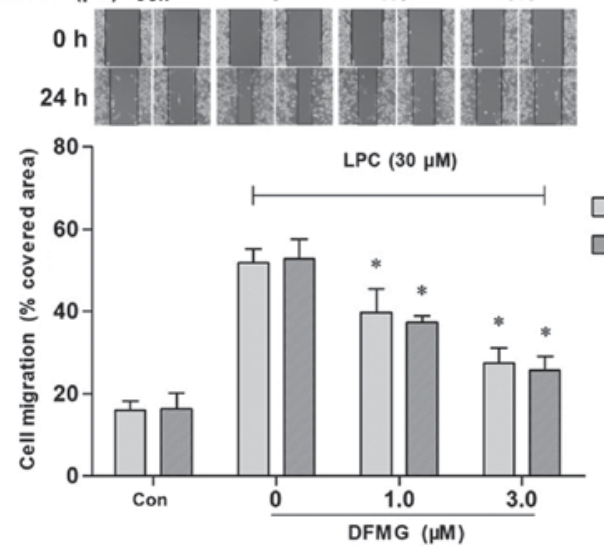

Figure 2. Effect of DFMG with different concentrations and pretreatment time periods on the proliferation and migration of VSMCs induced by co-culture with injured VECs. (A) DFMG at 1.0 and $3.0 \mu \mathrm{M}$ significantly inhibited the viability and migratory ability of VSMCs induced by co-culture with injured VECs. (B) The viability and migratory ability of VSMCs induced by co-culture with injured VECs were significantly decreased in VECs pretreated with DFMG $(1.0$ and $3.0 \mu \mathrm{M})$ for $12 \mathrm{~h}$. Wound healing experiment involving (C) VECs treated with different concentration of DFMG (D) for 12 or $24 \mathrm{~h}$. Data are presented as the mean \pm standard deviation of three independent experiments. ${ }^{*} \mathrm{P}<0.05$, vs. $0 \mu \mathrm{M}$ DFMG. ${ }^{*} \mathrm{P}<0.05$ vs. $0.3 \mu \mathrm{M}$ DFMG. LPC, lysophosphatidylcholine; DFMG, 7-difluoromethoxy-5,4'-dimethoxy-genistein; Con, control.

A

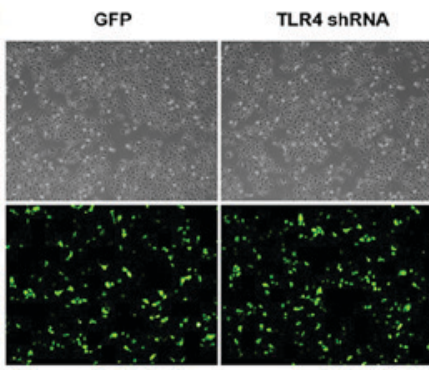

D

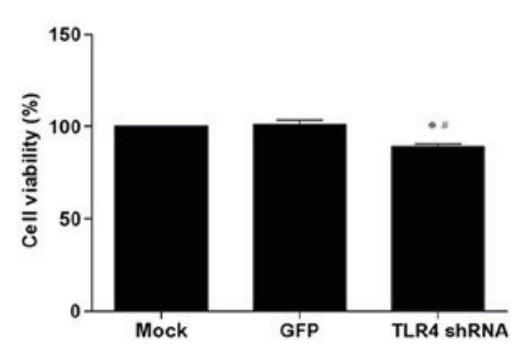

B

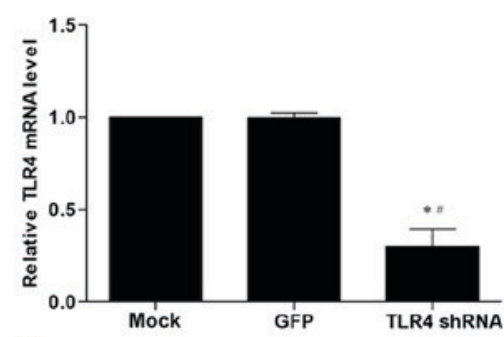

E

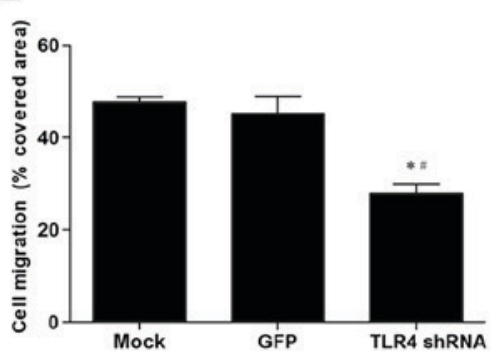

C

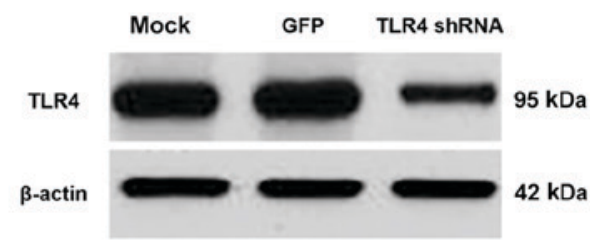

F

$\mathrm{Oh}$

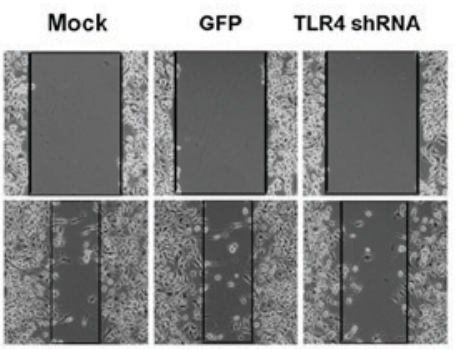

Figure 3. Effect of TLR4 shRNA transfection of VECs on the proliferation and migration of VSMCs induced by the co-culture model. (A) VECs transfected with GFP and TLR4 shRNA were captured under phase contrast and fluorescence microscopy. The expression of TLR4 (B) mRNA and (C) protein were markedly downregulated in the shRNA group. (D) viability and (E) migratory ability of VSMCs induced by the co-culture model were markedly decreased. (F) Wound healing experiment. Data are presented as the mean \pm standard deviation of three independent experiments. ${ }^{*} \mathrm{P}<0.05$ vs. mock. ${ }^{\#} \mathrm{P}<0.05 \mathrm{vs}$. GFP. TLR4, Toll-like receptor 4; shRNA, short hairpin RNA; GFP, green fluorescent protein empty vector.

factors lead to structural and functional alteration in the vascular endothelium, including lipid deposition into the intima, infiltration of inflammatory cytokines (IL-6, IL-8, TNF- $\alpha$ ), proliferation and migration of VSMCs, synthesis 
A

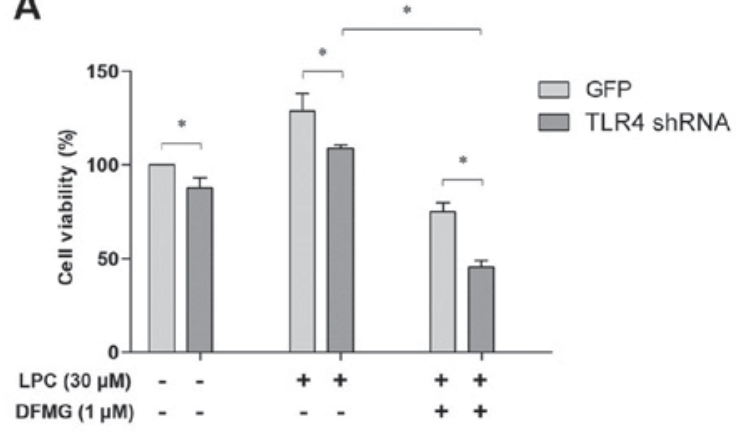

B

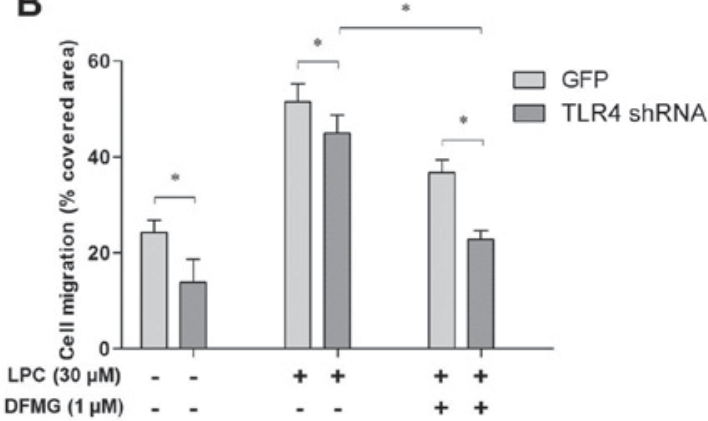

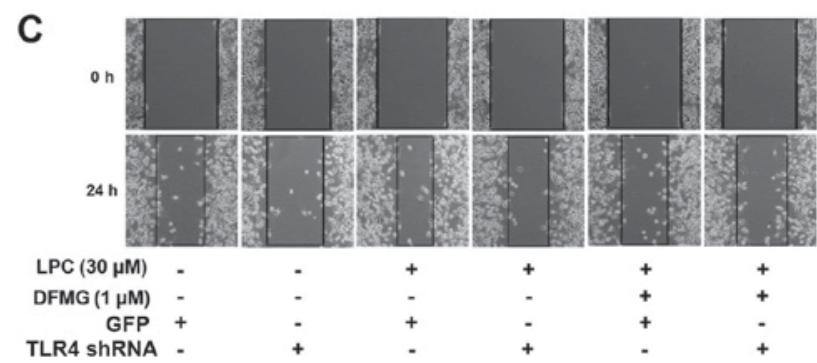

Figure 4. Combined effect of DFMG and TLR4 shRNA transfection of VECs on the proliferation and migration of VSMCs induced by co-culture with LPC-injured VECs. (A) Cell viability. (B) Cell migration. (C) Wound healing assay. Data are presented as the mean \pm standard deviation of three independent experiments. "P<0.05. LPC, lysophosphatidylcholine; DFMG, 7-difluoromethoxy-5,4'-dimethoxy-genistein; TLR4, Toll-like receptor 4; shRNA, short hairpin RNA.

A

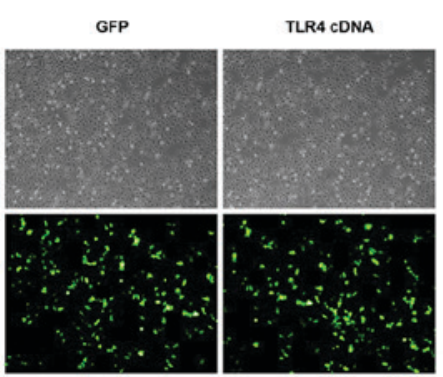

D

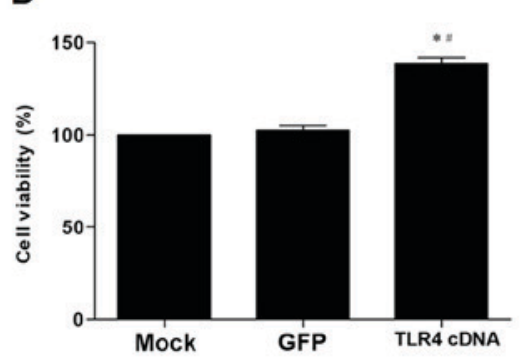

B

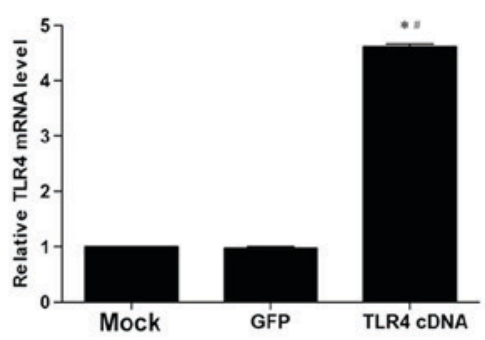

$\mathrm{E}$

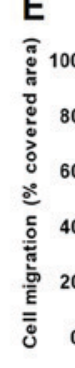

C

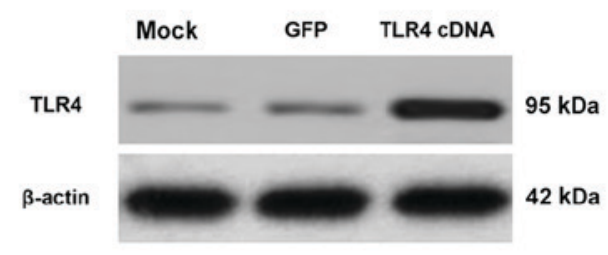

$\mathbf{F}$

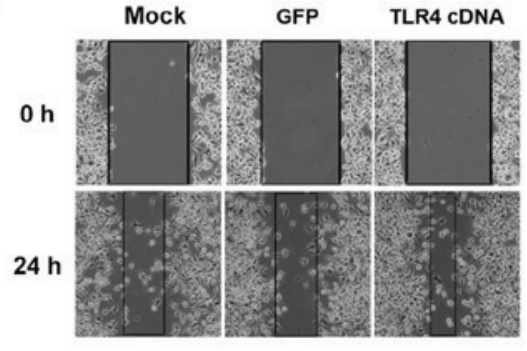

Figure 5. Effect of TLR4 cDNA transfection of VECs on proliferation and migration of VSMCs induced by the co-culture model. (A) VECs transfected with GFP and TLR4 cDNA were captured under phase contrast and fluorescence microscopy. Expression of TLR4 (B) mRNA and (C) protein were significantly upregulated. (D) Cell viability. (E) Cell migration. (F) Wound healing experiment. Data are presented as the mean \pm standard deviation of three independent experiments. ${ }^{\text {}} \mathrm{P}<0.05$ vs. the mock group. ${ }^{"} \mathrm{P}<0.05$ vs. the GFP group.

of extracellular matrix, and the formation of foam cells and plaques (30). Oxidation and enzymatic modification of low-density lipoproteins (LDL) leads to synthesis of LPC (31). LPC is a component of oxidized-LDL (ox-LDL) that can stimulate ox-LDL to cause AS (32). In the present study, LPC stimulated secretion of IL- 6 and TNF- $\alpha$ in VECs, and promoted the proliferation and migration of VSMCs in a co-culture model. These processes are crucial to neointima formation (33).
TLR4 is involved in the inflammatory reaction. Recently, the interaction between the TLR4 transduction pathway and AS has been extensively investigated (34-37). In the present study, a transient transfection was used in order to modulate the expression of TLR4 mRNA and protein in VECs. The results of the present study indicated that TLR4 cDNA promoted the proliferation and migration of VSMCs in a co-culture model, while TLR4 shRNA exerted the opposite effect. Furthermore, the results of the present study further supported the role of 
A

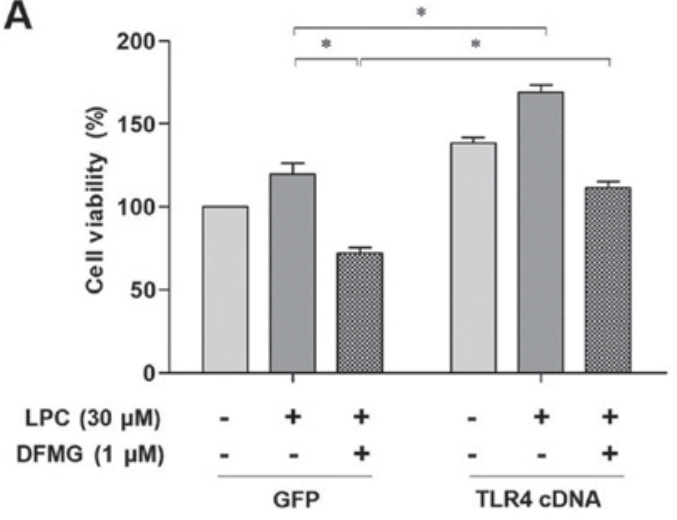

B

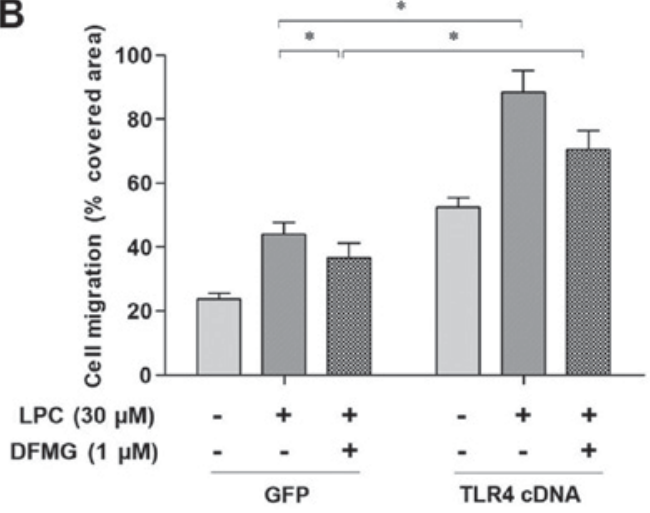

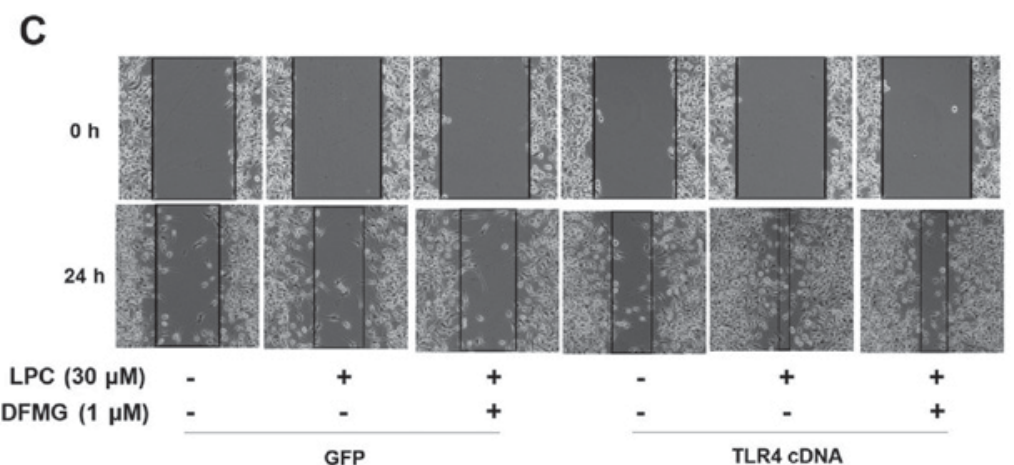

Figure 6. Combined effect of DFMG and TLR4 cDNA transfection of VEC on the proliferation and migration of VSMCs induced by co-culture with LPC-injured VECs. (A) Cell viability. (B) Cell migration. (C) Wound healing assay. Data are presented as the mean \pm standard deviation of three independent experiments. ${ }^{*} \mathrm{P}<0.05$.

TLR4 signaling in the proliferation and migration of VSMCs induced by co-culture with LPC-injured VECs.

Genistein, the lead compound of DFMG, is a natural isoflavone present in legumes and dentate plants. Genistein can alleviate inflammatory damage by altering TLR4/NF- $\mathrm{B}$ signaling $(22,23)$. In the present study, VECs were pretreated with DFMG prior to treatment with LPC and subsequently co-cultured with VSMCs. The results demonstrated that the proliferation and migration of VSMCs were decreased, which was dependent on the concentration of DFMG. In addition, the combination of treatment with DFMG and TLR4 shRNA transfection of VECs significantly suppressed the proliferation and migration of VSMCs induced by the co-culture with LPC-injured VECs. Inhibition of TLR4 signaling in VECs restrained abnormal proliferation and migration of VSMCs. TLR4 cDNA transfection of VECs enhanced proliferation and migration of VSMCs that were induced by co-culture with injured VECs, and attenuated the effects of DFMG.

In conclusion, the results of the present study demonstrated that DFMG effectively inhibited proliferation and migration of VSMCs induced by co-culture with LPC-injured VECs via regulation of the TLR4 signaling pathway. DFMG may be useful as a novel agent for the prevention of AS. However, the present study has certain limitations, including that the effects of DFMG on VSMCs have not been investigated in vivo. Furthermore, regulation of the TLR4/myeloid differentiation primary response 88 and TLR4/Toll-like receptor adaptor molecule 1 pathways by DFMG during this process has not been investigated. Consequently, understanding of detailed effects of DFMG on the TLR4 signaling pathway in AS requires further investigation.

\section{Acknowledgements}

Not applicable.

\section{Funding}

The present study was supported by a grant from the Natural Science Foundation of China (grant no. 81370382).

\section{Availability of data and materials}

All data generated or analyzed during this study are included in this published article.

\section{Authors' contributions}

XF designed the study and prepared the manuscript. LC and $\mathrm{HH}$ performed the experiments. $\mathrm{YZ}$ analysed the data. JC contributed to the interpretation of some of the data and revised the manuscript. All authors read and approved the final manuscript.

\section{Ethics approval and consent to participate}

Not applicable. 


\section{Consent for publication}

Not applicable.

\section{Competing interests}

The authors declare that they have no competing interests.

\section{References}

1. Heidari M, Mandato CA and Lehoux S: Vascular smooth muscle cell phenotypic modulation and the extracellular matrix. Artery Res 9: 14-18, 2015.

2. Lin X, He Y, Hou X, Zhang Z, Wang R and Wu Q: Endothelial cells can regulate smooth muscle cells in contractile phenotype through the miR-206/ARF6\&NCX1/exosome ax is. PLoS One 11: e0152959, 2016

3. Afzal TA, Luong LA, Chen D, Zhang C, Yang F, Chen Q, An W, Wilkes E, Yashiro K, Cutillas PR, et al: NCK associated protein 1 modulated by miRNA-214 determines vascular smooth muscle cell migration, proliferation, and neointima hyperplasia. J Am Heart Assoc 5: pii: e004629, 2016.

4. Ostriker A, Horita HN, Poczobutt J, Weiser-Evans MC and Nemenoff RA: Vascular smooth muscle cell-derived transforming growth factor- $\beta$ promotes maturation of activated, neointima lesion-like macrophages. Arterioscler Thromb Vasc Biol 34: 877-886, 2014.

5. Owens GK, Kumar MS and Wamhoff BR: Molecular regulation of vascular smooth muscle cell differentiation in development and disease. Physiol Rev 84: 767-801, 2004.

6. Gimbrone MA Jr and García-Cardeña G: Endothelial cell dysfunction and the pathobiology of atherosclerosis. Circ Res 118: 620-636, 2016.

7. Ding H, Li D, Zhang Y, Zhang T, Zhu H, Xu T, Luo Y and Wang C: Luteolin inhibits smooth muscle cell migration and proliferation by attenuating the production of Nox4, p-Akt and VEGF in endothelial cells. Curr Pharm Biotechnol 14: 1009-1015, 2014.

8. Xia Y, Bhattacharyya A, Roszell EE, Sandig M and Mequanint K: The role of endothelial cell-bound Jagged1 in Notch3-induced human coronary artery smooth muscle cell differentiation. Biomaterials 33: 2462-2472, 2012.

9. Zhou J, Li YS, Nguyen P, Wang KC, Weiss A, Kuo YC, Chiu JJ, Shyy JY and Chien S: Regulation of vascular smooth muscle cell turnover by endothelial cell-secreted microRNA-126: Role of shear stress. Circ Res 113: 40-51, 2013.

10. Li Y, Guo Y, Chen Y, Wang Y, You Y, Yang Q, Weng X, Li Q, Zhu X, Zhou B, et al: Establishment of an interleukin-1 $\beta$-induced inflammation-activated endothelial cell-smooth muscle cell-mononuclear cell co-culture model and evaluation of the anti-inflammatory effects of tanshinone IIA on atherosclerosis. Mol Med Rep 12: 1665-1676, 2015.

11. Satoh S, Yada R, Inoue H, Omura S, Ejima E, Mori T, Takenaka K, Kawamura N, Numaguchi K, Mori E, et al: Toll-like receptor-4 is upregulated in plaque debris of patients with acute coronary syndrome more than Toll-like receptor-2. Heart Vessels 31: 1-5, 2016.

12. Roshan MH, Tambo A and Pace NP: The role of TLR2, TLR4, and TLR9 in the pathogenesis of atherosclerosis. Int J Inflam 2016 $1532832,2016$.

13. Lim S and Park S: Role of vascular smooth muscle cell in the inflammation of atherosclerosis. BMB Rep 47: 1-7, 2014.

14. Xing S, Zheng F, Zhang W, Wang D and Xing Q: Relationship between toll-like receptor 4 levels in aorta and severity of atherosclerosis. J Int Med Res 42: 958-965, 2014.

15. Kapelouzou A, Giaglis S, Peroulis M, Katsimpoulas M, Moustardas P, Aravanis CV, Kostakis A, Karayannakos PE and Cokkinos DV: Overexpression of toll-like receptors 2, 3, 4 and 8 is correlated to the vascular atherosclerotic process in the hyperlipidemic rabbit model: The effect of statin treatment. J Vasc Res 54: 156-169, 2017.

16. Tang YL, Jiang JH, Wang S, Liu Z, Tang XQ, Peng J, Yang YZ and Gu HF: TLR4/NF- $\mathrm{KB}$ signaling contributes to chronic unpredictable mild stress-induced atherosclerosis in ApoE-/mice. PLoS One 10: e0123685, 2015.

17. Pahwa R, Nallasamy P and Jialal I: Toll-like receptors 2 and 4 mediate hyperglycemia induced macrovascular aortic endothelial cell inflammation and perturbation of the endothelial glycocalyx. J Diabetes Complications 30: 563-572, 2016.
18. Wang XQ, Wan HQ, Wei XJ, Zhang Y and Qu P: CLI-095 decreases atherosclerosis by modulating foam cell formation in apolipoprotein E-deficient mice. Mol Med Rep 14: 49-56, 2016.

19. Yang X, Coriolan D, Murthy V, Schultz K, Golenbock DT and Beasley D: Proinflammatory phenotype of vascular smooth muscle cells: Role of efficient Toll-like receptor 4 signaling. Am J Physiol Heart Circ Physiol 289: H1069-H1076, 2005.

20. Jiang D, Li D, Cao L, Wang L, Zhu S, Xu T, Wang C and Pan D: Positive feedback regulation of proliferation in vascular smooth muscle cells stimulated by lipopolysaccharide is mediated through the TLR 4/Rac1/Akt pathway. PLoS One 9: e92398, 2014.

21. $\mathrm{Li} \mathrm{H}, \mathrm{Xu} \mathrm{H}$ and Liu S: Toll-like receptors 4 induces expression of matrix metalloproteinase-9 in human aortic smooth muscle cells. Mol Biol Rep 38: 1419-1423, 2011.

22. Zhou X, Yuan L, Zhao X, Hou C, Ma W, Yu H and Xiao R: Genistein antagonizes inflammatory damage induced by $\beta$-amyloid peptide in microglia through TLR4 and NF- $\kappa$ B. Nutrition 30: 90-95, 2014.

23. Ma W, Ding B, Yu H, Yuan L, Xi Y and Xiao R: Genistein alleviates $\beta$-amyloid-induced inflammatory damage through regulating Toll-like receptor 4/nuclear factor $\kappa B$. J Med Food 18: 273-279, 2015.

24. Liu F, Cao JG, Li C, Tan JS and Fu XH: Protective effects of 7-difluoromethyl-5,4'-dimethoxygenistein against human aorta endothelial injury caused by lysophosphatidyl choline. Mol Cell Biochem 363: 147-155, 2012

25. Liu S, Li L, Zhang J, Huang H, Yang S, Ren C, Fu X and Zhang Y: 7-difluoromethyl-5, 4'-dimethoxygenistein reverses LPC-induced apoptosis of HUVE-12 cells through regulating mitochondrial apoptosis pathway. Curr Signal Transd Ther 9: 50-58, 2014.

26. Zhang Y, Li L, You J, Cao J and Fu X: Effect of 7-difluoromethyl-5, 4'-dimethoxygenistein on aorta atherosclerosis in hyperlipidemia ApoE(-/-) mice induced by a cholesterol-rich diet. Drug Des Devel Ther 7: 233-242, 2013.

27. Fu XH, Wang L, Zhao H, Xiang HL and Cao JG: Synthesis of genistein derivatives and determination of their protective effects against vascular endothelial cell damages caused by hydrogen peroxide. Bioorg Med Chem Lett 18: 513-517, 2008

28. Chen L, DeWispelaere A, Dastvan F, Osborne WR, Blechner C, Windhorst S and Daum G: Smooth muscle-alpha actin inhibits vascular smooth muscle cell proliferation and migration by inhibiting Rac1 activity. PLoS One 11: e0155726, 2016.

29. Livak KJ and Schmittgen TD: Analysis of relative gene expression data using real-time quantitative PCR and the 2(-Delta Delta C(T)) method. Methods 25: 402-408, 2001.

30. Cibor D, Domagala-Rodacka R, Rodacki T, Jurczyszyn A, Mach T and Owczarek D: Endothelial dysfunction in inflammatory bowel diseases: Pathogenesis, assessment and implications. World J Gastroenterol 22: 1067-1077, 2016.

31. Sevastou I, Kaffe E, Mouratis MA and Aidinis V: Lysoglycerophospholipids in chronic inflammatory disorders: The PLA(2)/LPC and ATX/LPA axes. Biochim Biophys Acta 1831: 42-60, 2013

32. Voight BF, Peloso GM, Orho-Melander M, Frikke-Schmidt R, Barbalic M, Jensen MK, Hindy G, Hólm H, Ding EL, Johnson T, et al: Plasma HDL cholesterol and risk of myocardial infarction: A mendelian randomisation study. Lancet 380: 572-580, 2012.

33. Wang X, Hu G, Gao X, Wang Y, Zhang W, Harmon EY, Zhi X, Xu Z, Lennartz MR, Barroso M, et al: The induction of yes-associated protein expression after arterial injury is crucial for smooth muscle phenotypic modulation and neointima formation. Arterioscler Thromb Vasc Biol 32: 2662-2669, 2012.

34. Luo H, Wang J, Qiao C, Ma N, Liu D and Zhang W: Pycnogenol attenuates atherosclerosis by regulating lipid metabolism through the TLR4-NF-кB pathway. Exp Mol Med 47: e191, 2015.

35. Lee GL, Wu JY, Tsai CS, Lin CY, Tsai YT, Lin CS, Wang YF, Yet SF, Hsu YJ and Kuo CC: TLR4-activated MAPK-IL-6 axis regulates vascular smooth muscle cell function. Int J Mol Sci 17: pii: E1394, 2016.

36. Hosseini H, Li Y, Kanellakis P, Tay C, Cao A, Liu E, Peter K, Tipping P, Toh BH, Bobik A and Kyaw T: Toll-like receptor (TLR)4 and MyD88 are essential for atheroprotection by peritoneal B1a B cells. J Am Heart Assoc 5: pii: e002947, 2016.

37. Ding Y, Subramanian S, Montes VN, Goodspeed L, Wang S, Han C, Teresa AS III, Kim J, O'Brien KD and Chait A: Toll-like receptor 4 deficiency decreases atherosclerosis but does not protect against inflammation in obese low-density lipoprotein receptor deficient mice. Arterioscler Thromb Vasc Biol 32: 1596-1604, 2012.

This work is licensed under a Creative Commons

Attribution-NonCommercial-NoDerivatives 4.0 International (CC BY-NC-ND 4.0) License. 